\section{Alexander Meng}

\section{Betrachtung nach der modernen Medizin}

Um die Möglichkeiten der Akupunktur bei Allergien besser zu verstehen, ist erst einmal das Krankheitsbild aus der modernen Medizin in Erinnerung zu bringen. Eine allergische Erkrankung entsteht verzögert oder sofort nach Kontakt der Schleimhaut bzw. der Körperoberfläche mit Allergenen. Wir sehen diese Reaktionen an der Haut, der Nase, der Lunge, den Augen und im Magen-Darm-Trakt. Diese treten nach Kontakt bzw. Inhalation von saisonalen Pollen von Sträuchern, Bäumen oder Gräsern auf. Ganzjährig werden sie durch Tierhaare, Schimmelpilze bzw. verschiedene Chemikalien, aber auch nach Kontakt mit bzw. Verzehr von Meeresfrüchten, Obst, Gemüse, Nahrungsmitteln und bestimmten Arzneimitteln usw. verursacht.

Die Schwellung und Hyperämisierung der Nasenschleimhaut gibt das Bild der Rhinitis allergica. Das Bild kann aber auch durch einen unspezifischen Stimulus ausgelöst werden; dann sprechen wir von Rhinitis vasomotorica. Allgemeine therapeutische Massnahmen wie unter anderem Prävention, Allergenkarenz, spezifische Hyposensibilisierung, Antihistaminika und intranasale Aspiration von Glukokortikoiden werden angewendet. Die erste Spitalsambulanz für Akupunktur wurde 1956 durch

\title{
Akupunktur bei Allergien
}

Der unterschiedliche Zugang von Traditioneller Chinesischer Medizin (TCM) und moderner Medizin zu Diagnostik und Therapie ermöglicht eine Erweiterung des ärztlichen Handelns. Es wird zu Beginn 2-mal wöchentlich behandelt und nach 6 Behandlungen dann auf 1-mal wöchentlich reduziert. Insgesamt wird in der Regel nach 12 Sitzungen eine Behandlungspause von 3 bis 6 Monaten eingelegt. Allgemein sollte die Akupunktur bei saisonalen Allergien 2-4 Wochen zuvor beginnen.

Johannes Bischko an der HNO-Abteilung in der Wiener Poliklinik initiiert. Der Therapieerfolg bei Rhinitis vasomotorica war so unbestritten, dass viele HNO-Ärzte die Akupunktur in ihrer Praxis anwendeten.

\section{Was kann die Akupunktur \\ leisten und wie versteht \\ die TCM die Allergie?}

Akupunktur, Ernährung, Bewegungstherapie (Qigong, Taijiquan), Tuina, Akupressur, Moxibustion usw. können hier sinnvolle Dienste leisten. Die Dauermedikation und die Bedarfsmedikation müssen die Patienten weiter nehmen.

TCM ist Wellness und die Befolgung des Lebens im Rhythmus der Natur, d.h. nach der Jahres- und Tageszeit. Die TCM legt grossen Wert auf die harmonische Einheit zwischen Leib, Bewusstsein und Unterbewusstsein. Dann kann der Mensch mit seinem sozialen Umfeld in Einklang leben. Die Prävention ist von größerer Bedeutung als die Therapie chronischer bzw. akuter Erkrankungen.

Unterschiede in der Anatomie in der TCM im Vergleich mit der modernen Medizin sehen wir im Makroskopischen und in der Physiologie und Ätiologie einer Störung. Denn bei der TCM erfolgt dies alles ohne morphologische Zuordnung; die Befunde werden in Analogie zum Universum
(Bilder) analysiert, genauer gesagt intuitiv vermutet. Hingebend bemüht sich die moderne Medizin um eine genaue mikroskopische Morphologie (Läsion) bei der Zuordnung der Störungen.

Aus der Sicht der TCM stellt das Krankheitsbild bei Allergien folgende Strukturen in Wechselbeziehung: Nase $\leftrightarrow$ Lunge $\leftrightarrow$ Haut $\leftrightarrow$ (Magen und) Darm.

Nachfolgend das Erklärungsmodell aus der modernen Medizin: Unter der Beziehung «Nase zu Lunge» verstehen wir, dass die Nase zu den oberen Atemwegen gehört; «Haut zu Lunge» und «Haut zu Magen und Darm» können wir als einen kutiviszeralen $\mathrm{Re}$ flexweg plausibel machen. Deshalb kann ein therapeutischer Nadelreiz auch eine Antwort an inneren Organen auslösen. Wenn jemand ferner eine allergische Reaktion aufweist, können sich sowohl Symptome an der Körperoberfläche als auch im MagenDarm-Trakt zeigen. Klinische Zeichen können eine laufende Nase im Frühling, Augenjucken, Nahrungsunverträglichkeit, Durchfälle, MagenDarm-Krämpfe usw. sein.

\section{Differentialdiagnose in der TCM und die «Dreier Regel»}

Der unterschiedliche Zugang von TCM und moderner Medizin zu Diagnostik und Therapie ermöglicht

\section{KARGER}

Fax +497614520714 Information@Karger.de www.karger.com
(๑) 2010 S. Karger GmbH, Freiburg

Accessible online at: www.karger.com/sz
Prof. Dr. med. univ. Alexander Meng

Facharzt für Neurologie/Psychiatrie

Frauenfelder Strasse 8, 1170 Wien, Österreich

Tel. +4314861277

alexander@meng.at 
eine Erweiterung des ärztlichen Handelns. Die nicht apparative und nicht invasive Untersuchung der TCM erlaubt einen Funktionsstatus ohne exakte Zuordnung zu einem morphologischen Substrat. Um dem Therapeuten die Frage nach den am besten geeigneten Akupunkturpunkten zu erleichtern, wurde von mir die «Dreier Regel der Wiener Schule» entwickelt. Die ersten beiden Fragen beziehen sich auf den Ort der Erkrankung: «Welcher Meridian» bzw. "welches Organ ist betroffen». Die dritte Frage berücksichtigt die sog. Modalitäten, die einerseits Zuordnungen zu «Funktionskreisen» erlauben, andererseits den Akupunkteur bis zur chinesischen Syndromlehre führen können.

Die Medizintheorie der TCM basiert in erster Linie auf empirischen Erfahrungen. Im ältesten Lehrbuch der TCM, dem «Neijing», das schon vor über 2000 Jahren publiziert wurde, finden wir Abhandlungen über Anatomie, Physiologie, Pathophysiologie, Ätiologie, Diagnostik, Therapie und Prophylaxe. Die Lehre von Yin und Yang, von den 5 Elementen und von den Meridianen sowie die Organlehre stellen einen Teil der Haupttheorien der TCM dar (Abb. 1, 2). Im Gegensatz dazu wurzelt die europäische Medizin in der Weltanschauung der alten Griechen, die das Atom als Bestandteil der Materie gesehen hatten. Die exakte und fortwährend verfeinerte Strukturforschung (Grundlagenforschung der Anatomie, Immunologie, Gentechnik usw.) wurde stets in Verbindung mit der Funktion gebracht. In der modernen Medizin
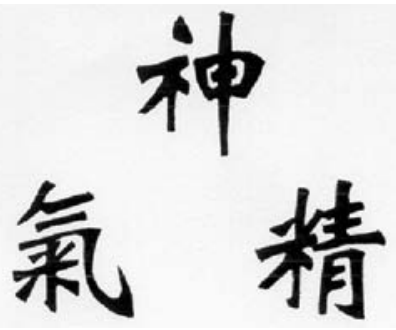

Abb. 1. Die Drei Schätze der Gesundheit: Geist-Shen, Vitalenergie-Qi und Essenz der Niere-Jing (aus Meng A: Skriptum Österreichischer Arbeitskreis für Tuina).

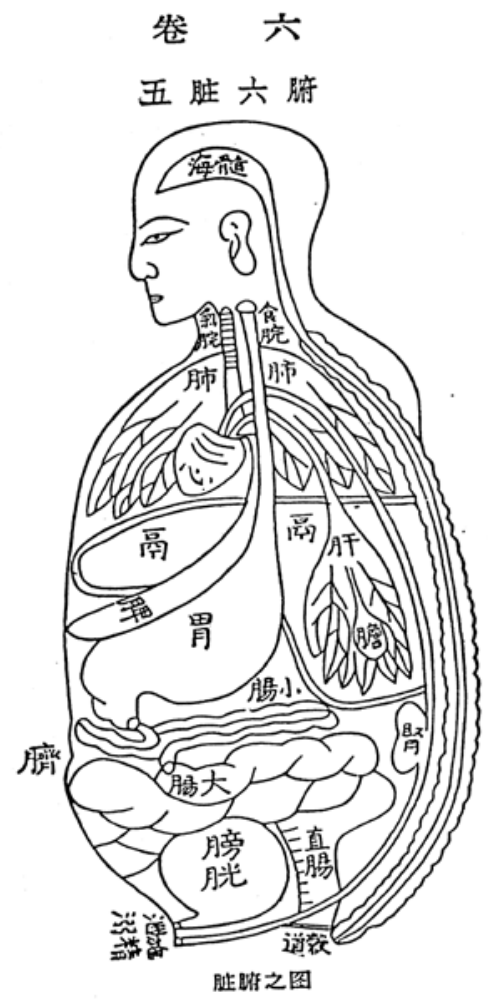

Abb. 2. Organbild aus China, 1601 (aus Meng A, Exel W: Chinesische Heilkunst. Wien, Kneipp, 2005).

bedarfjede Funktion bzw. jeder Funktionsverlust und jede Funktionswiederherstellung einer strukturellen Untermauerung (d.h. Reparaturmedizin). Im alten China wurde das Modell Qi als Bestandteil des Universums und des Menschen gesehen. Die TCM legt im Gegensatz zur modernen Medizin höchsten Wert aufSinnerfassung (Deyi), wobei auf Erscheinungsbilder verzichtet wird (Wangxiang). Eine Schwäche der TCM liegt jedoch darin, dass zwar jedem Organ wichtige physiologische Aufgaben zukommen, aber über das morphologische Substrat nur sehr vage Vorstellungen existieren. Oberflächlich betrachtet könnte man z.B. das Herz mit seiner Funktion als Blutpumpe in der modernen Medizin und in der TCM gleichsetzen. Die TCM schreibt dem Herzen aber noch weitere wichtige Aufgaben $\mathrm{zu}$, die in der modernen Medizin dem Nervensystem und dem Gehirn zugeordnet werden. Zur Vereinfachung der komplexen Syndrome der TCM wurde vom Autor Ende der 1970er-Jahre erstmals die «Dreier Re- gel der Akupunktur» formuliert, um dem Akupunkturanfänger im Westen die chinesische Reflextherapie zugänglich zu machen [1].

\section{Einige Empfehlungen für gut ansprechende Indikationen aus der eigenen Akupunkturpraxis}

Die Behandlung wird zu Beginn 2-mal wöchentlich vorgenommen und nach 6 Behandlungen dann auf 1-mal wöchentlich reduziert. Insgesamt wird in der Regel nach 12 Sitzungen eine Behandlungspause von 3 bis 6 Monaten eingelegt. Allgemein soll die Akupunktur bei saisonalen Allergien 2-4 Wochen zuvor beginnen. Wenn der Patient jedoch bereits Symptome zeigt, kann die eine rasche Abschwellung bewirkende Akupunktur den Patienten schon bei der ersten Behandlung sehr positiv überraschen. Eine solche «Kontrollierte, randomisierte, einfach blinde Studie zur Wirksamkeit einer einmaligen augmentierten Akupunkturbehandlung bei akuter Rhinitis allergica» hat Cornelius Lenz von der Medizinischen Fakultät der Albert-Ludwigs-Universität Freiburg im Breisgau 2008 in seiner Doktorarbeit vorgelegt [2].

Bewährt haben sich in meiner Praxis folgende Behandlungsschemata mit individueller Ergänzung, meist nach der vereinfachten Differentialdiagnose der Dreier Regel der Wiener Schule, d.h. mit den zusätzlichen Punkten Meridiansyndrom und Organsyndrom sowie Modalitäten wie die 8 Prinzipien, die Fünf-Elemente-Lehre usw.

\section{Chronische Rhinitis}

PdM (in der Mitte zwischen den Augenbrauen), Di 4 (Mitte der Metacarpale 2, radiale Seite), Di 20 (in der Höhe des Nasenlochs, $2 \mathrm{~mm}$ lateral), G 20 (knapp distal und medial vom Processus mastoideus).

\section{Chronische Sinusitis}

Di 20, G 14 (ein Daumenbreit kranial von der Augenbrauenmitte), B 2 (am 
medialen Ende der Augenbrauen), G 20, Di 4, Lu 7 (palmar, drei Querfinger über dem radialen Ende der Handgelenksquerfalte), Le 2 (dorsal, knapp $5 \mathrm{~mm}$ über der Schwimmhautfalte zwischen der 2. und 3. Zehe).

Zusätzliche Punkte je nach Syndromen im Sinne der bioklimatischen Ätiologie:

- Wind/Kälte/Hitze: B 13 (Zustimmungspunkt der Lunge, zwei Querfinger lateral vom 3. Brustwirbeldornfortsatz), Lu 7, Di 4;

- Wind/Kälte: B 11 (zwei Querfinger lateral vom 1. Brustwirbeldornfortsatz), B 12 (zwei Querfinger lateral vom 2. Brustwirbeldornfortsatz, hier Nadeln, Moxibustion bzw. Schröpfen);

- Wind/Hitze: Di 11 (am radialen Ende der Ellenbogenbeugefalte), LG 14 (distal vom 7. Halswirbeldornfortsatz).

Wenn klinisch auch Organbeteiligungen vorliegen:

- Viel Schleim/Lunge: B 13, Lu 9 (palmar, am radialen Ende der Handgelenksquerfurche), MP 3 (knapp proximal vom Grosszehengrundgelenk), M 40 (auf gleicher Höhe wie M 38, aber ein Daumenbreit lateral; Streckenmitte zwischen höchstem Punkt des Malleolus externus und dem Kniegelenkspalt), Di 4;

- Leber-Feuer/Lunge: B 13, Lu 10 (palmar, Mitte von Thenar), Le 2, G 34 (distal und medial vom Fibulaköpfchen);

- Yin-Leere/Lunge-Niere: B 13, B 38 (vier Querfinger lateral vom 3. Brustwirbeldornfortsatz), Lu 5 (Beugeseite des Ellbogens, radial von der Bizepssehne), N 6 (distal vom $\mathrm{M}$. internus).

\section{Asthma bronchiale}

Voller Typ:

- Dingchuan (ein Daumenbreit lateral von LG 14), Lu 7, Lu 5, Di 4, KG 17 (am Sternum, in Höhe des 4. Intercostalraums (ICR));

- Wind-Kälte: B 12, B 13;

- Wind-Hitze: Di 11, LG 14;
- Leber-Qi-Stagnation: Le 3 (auf dem Fussrücken, im proximalen Winkel zwischen Metatarsale II und III), Le 14 (Medioclavicularlinie, 6. ICR, direkt unter der Mamilla), MP 3;

- viel Schleim: KG 12 (in der Mitte zwischen Nabel und Xiphoid), M 40;

- Brustschmerzen: Lu 1 (zwei Daumenbreit unter der Unterkante der Clavicula, 1. ICR, zwei Handbreit lateral der Medianen), KG 21 (auf dem Sternum in Höhe des Oberrandes der 1. Rippe).

Leerer Typ:

- LG 14, Dingchuan (EX-B1, von C7 ein Fingerbreit, lateral), B 13, B 38, Lu 9, M 36 (ein Querfinger lateral der vorderen Tibiakante, zwei Querfinger unterhalb des Unterrandes des Fibulaköpfchens);

- Milz-Leere: B 20 (Zustimmungspunkt von MP, zwei Fingerbreit lateral vom Dornfortsatz des 11. Brustwirbels), B 21 (Zustimmungspunkt von Magen, zwei Fingerbreit lateral vom Dornfortsatz des 12. Brustwirbels), MP 3;

- Lunge-Niere-Leere: B 23 (Zustimmungspunkt von Niere, zwei Fingerbreit des 2. Dornfortsatzes des 2. Lendenwirbels), KG 6 (zwei Querfinger unterhalb des Nabels), N 3 (zwischen stärkster Vorwölbung des $M$. medialis und der Achillessehne).

\section{Prophylaxe des Asthma bronchiale}

Die beste Zeit für die «Prophylaxe» von Asthma bronchiale [3] ist der Sommer mit Nadelakupunktur bzw. zusätzlichem Schröpfen:

- KG 21 (Nadeln gleich entfernen), LG 14, Parav. Th. 2-3,

- viel Schleim: M 40;

- Husten/Keuchen: Lu 7, B 23, MP 6 (vier Querfinger oberhalb der grössten Zirkumferenz des Innenknöchels, am Hinterrand der Tibia), N 3. Am besten noch zusätzlich die Punkte 30 min schwach elektrisch stimulieren, danach etwa 5 min schröpfen.

\section{Allgemeine Aktivierung} des Immunsystems

Moxibustion (tägliche lokale Wärmebehandlung, bis zur lokalen Rötung, pro Punkt etwa $5 \mathrm{~min}$, pro Sitzung etwa 6 Punkte):

- Hauptpunkte: LG 14, Di 11, M 36, KG 6, B 38, B 23.

- Zusätzliche Punkte: B 18, B 20, MP 6, MP 10 (bei gebeugtem Knie zwei Daumenbreit oberhalb des Patellaoberrandes).

Professor Stefan Willich und PD Dr. Benno Brinkhaus, Forscher der Charité-Universitätsmedizin Berlin, haben in einer Studie mit mehr als 5000 Pollenallergikern herausfinden können, dass Akupunktur Beschwerden bei Heuschnupfen lindert. Die Ergebnisse wurden in der Fachzeitschrift Annals of Allergy, Asthma and Immunology veröffentlicht [4]: "Akupunktur bessert bei bis zu 80 Prozent aller Patienten die Heuschnupfenbeschwerden, zusätzlich steigert sich die Lebensqualität der Patienten deutlich.» Daraus schliessen die Wissenschaftler, dass Akupunktur in der Routineversorgung wirkt. «Uns hat besonders beeindruckt, dass sich bei Heuschnupfen ähnliche Ergebnisse zeigen wie bei chronischen Schmerzerkrankungen», sagt Prof. Willich.

Wie genau aber wirkt die Akupunktur bei Heuschnupfen? Diese Frage interessiert uns sicherlich alle als Ärzte.

\section{Literatur}

1 Meng A: Die «Dreier Regel» für die Akupunktur und Tuina im Westen. Dtsch Z Akupunkt 2005;48:31-33.

2 Lenz C: Kontrollierte, randomisierte, einfach blinde Studie zur Wirksamkeit einer einmaligen augmentierten Akupunkturbehandlung bei akuter Rhinitis allergica. Inaugural-Dissertation. Giessen, VVB Laufersweiler, 2008.

3 Meng A, Exel W: Chinesische Heilkunst. Wien, Kneipp, 2005, pp 100-114.

4 Brinkhaus B, et al: Acupuncture in patients with allergic rhinitis: a pragmatic randomized trial. Ann Allergy Asthma Immunol 2008;101:535543. 